\title{
Awareness of the cost of psychotropic medication among doctors: a service evaluation
}

\author{
Dhananjay Kumar Singh, ${ }^{1}$ Shakil Khawaja, ${ }^{2}$ Ishaq Pala, ${ }^{1}$ Jaleel Khaja, ${ }^{3}$ Ray Krishnanu, ${ }^{1}$ \\ Heather Walker, ${ }^{2}$ Julian Bustin ${ }^{1}$
}

The Psychiatrist (2010), 34, 364-366, doi: 10.1192/pb.bp.109.026914

${ }^{1}$ Barts and the London Training Scheme; ${ }^{2}$ North East London Foundation Trust; ${ }^{3}$ South London and Maudsley NHS Foundation Trust, London, UK

Correspondence to Julian Bustin (julianbustin@gmail.com)

\begin{abstract}
Aims and method Cost-effective prescribing is an increasingly important aspect of our practice. A service evaluation was carried out to assess the level of awareness and knowledge of different aspects of cost-effective prescribing among doctors working in the North East London Foundation Trust. A semi-structured questionnaire was used to benchmark knowledge against six standards.
\end{abstract}

Results The survey was completed by $71 \%$ of doctors working in adult or old age psychiatry. A total of $2 \%$ of doctors stated that they should always take into consideration the price of the drug when prescribing and only $5 \%$ of doctors claimed to know the price of medications they prescribe most frequently.

Clinical implications Strategies to improve the poor level of knowledge and awareness in this area of clinical practice would be of benefit in making the best use of limited financial resources without any detriment to patient care.

Declaration of interest None.
The National Audit Office stated that the National Health Service (NHS) in England could save more than $£ 300$ million a year by being more efficient when prescribing drugs. They added that general practitioners (GPs) could make more use of cheaper and non-brand versions of the common prescription drugs without harming care. ${ }^{1}$ The cost of drugs is one of the most important aspects of costeffective prescribing that has become increasingly important for the NHS and foundation trusts due to their limited resources. This is also reflected in recent National Institute for Health and Clinical Excellence (NICE) health technology appraisals. ${ }^{2-4}$ Also, the British National Formulary (BNF) states that 'where there is a choice of suitable preparations for a particular disease, the relative cost may be used in making a selection'. ${ }^{5}$ Consequently, doctors are becoming increasingly responsible for cost-effective prescribing.

This service evaluation explored whether it is necessary to increase, among psychiatrists in the North East London Foundation Trust, the level of awareness and knowledge in this area of clinical practice. The following six standards relating to cost-effective prescribing were agreed upon by the service-evaluation team based on multidisciplinary discussions, previous experience of the North East London Foundation Trust Drugs and Therapeutics Committee, NICE guidelines, ${ }^{2-4}$ Prescribing Costs in Primary Care ${ }^{1}$ and the BNF. ${ }^{5}$

1 Doctors should always prescribe generically rather than by trade name.

†See commentaries, pp. 367-368 and pp. 369-370, this issue.
2 Doctors should always take into consideration the price of the drug when prescribing.

3 Doctors should sometimes change their prescribing practice according to the price of the drug.

4 All doctors should be aware of at least one method of accessing the price of medications they prescribe.

5 All doctors should know at least one process/system in the Trust to promote cost-effective prescribing.

6 All doctors should be able to rank two or three drugs that they prescribe most frequently from each psychotropic group according to price.

\section{Method}

The study was carried out in the North East London Foundation Trust with the Trust's approval. A list of all doctors, across all grades in general adult and old age psychiatry, working in the Trust between November 2007 and January 2008 was compiled. All doctors were approached by a member of the service-evaluation team and, those who consented, completed the survey. The service-evaluation instrument was a semi-structured questionnaire designed by the team incorporating the six standards outlined above and was based on previous work published by Bustin et al. ${ }^{6}$

In the first part of the evaluation instrument, doctors were asked their grade, specialty, how often they considered the price of medications when prescribing, whether the price of a drug would change their prescribing practice, whether they use generic or trade names, whether they have 
ready access to the price of medications within the hospital and whether they know of any process or systems in the Trust to promote cost-effective prescribing. In the second part, doctors were asked to pick, from each pharmacological group in the BNF $54,{ }^{5}$ three psychotropic medications that they most frequently prescribe. In the last part, they were required to rank the selected drugs from each group in decreasing order of price, indicate whether they know the price for each of these and, for the dosage and form they most commonly prescribe, estimate the lowest price for 4 weeks of treatment with each of the selected drugs (serviceevaluation instrument available from the author on request).

The data were compared with prices in the BNF $54^{5}$ and were analysed using Access XP for Windows.

\section{Results}

A total of $71 \%(102 / 143)$ of the doctors in the Trust completed the service evaluation. Table 1 gives the distribution by grade of all the doctors who participated in the service evaluation. Five of the six standards were achieved by less than $50 \%$ of doctors. Only standard three was achieved by $70 \%$ of doctors.

A total of $44 \%$ of doctors always prescribed generically and $87 \%$ of doctors prescribed generically on a regular basis. Interestingly, $33 \%$ of doctors admitted to never taking into consideration the price of the drug when prescribing (Fig. 1) and $17 \%$ admitted to never changing their prescribing practice according to the price of the drug (Fig. 2).

Only $5 \%$ of doctors claimed to know the prices of medications they prescribe most frequently. Of these, a total of $17 \%$ could estimate the prices within $20 \%$ above or below the actual cost. Barely $23 \%$ of doctors were able to rank two or three drugs that they prescribed frequently according to price. In estimating the prices of drugs, doctors were most successful with antidepressants (40\%). The percentage for antipsychotics, anxiolytics, hypnotics, antimanic and drugs for dementia were $23 \%, 30 \%, 21 \%, 24 \%$ and $11 \%$ respectively. Overpricing clozapine, haloperidol, selective serotonin reuptake inhibitors (SSRIs) and lithium while underpricing quetiapine were common mistakes.

A total of $35 \%$ of doctors stated that they had, within their hospitals, ready access to the cost of the drugs they

\begin{tabular}{|lcr|}
\hline Table 1 & \multicolumn{3}{l}{$\begin{array}{l}\text { Distribution by grade of all the doctors who } \\
\text { participated in the service evaluation }\end{array}$} \\
$\begin{array}{lcc}\text { Total sample } \\
n\end{array}$ & $\begin{array}{c}\text { Responded } \\
n(\%)\end{array}$ \\
\hline Doctors, all grades & 143 & $102(71)$ \\
\hline Consultants & 50 & $27(54)$ \\
\hline $\begin{array}{l}\text { Specialty registrar/specialist } \\
\text { trainee (ST4-6) }\end{array}$ & 15 & $13(87)$ \\
\hline Specialist trainee (ST1-ST3) & 38 & $35(92)$ \\
\hline General practitioner trainees & 12 & $10(83)$ \\
\hline Associate specialists & 7 & $3(43)$ \\
\hline Staff grades & 16 & $11(69)$ \\
\hline Foundation year trainees (FY1-2) & 5 & $3(60)$ \\
\hline
\end{tabular}

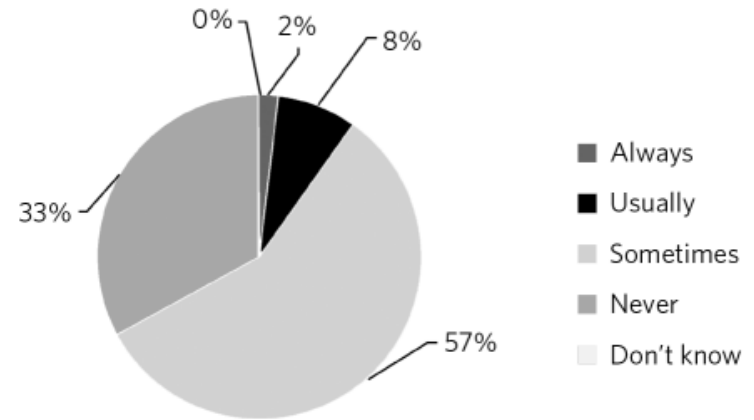

Fig 1 Doctors who take into consideration the price of the drug when prescribing.

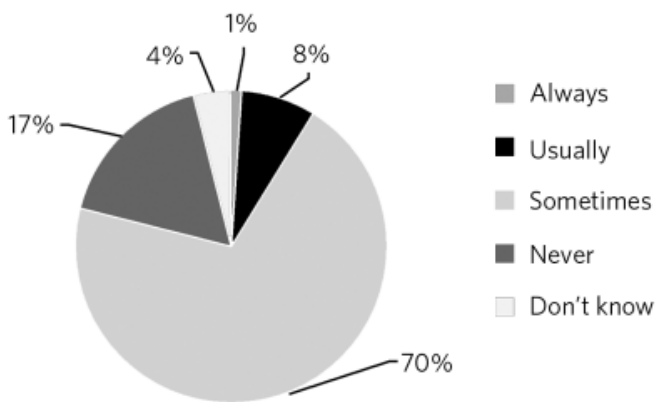

Fig 2 Doctors who change their prescribing practice according to the price of the drug.

prescribe. Of this group, $70 \%$ mentioned having access to the BNF and $20 \%$ mentioned having access to pharmacists. The remaining $10 \%$ reported using other methods such as the internet. Only $34 \%$ knew of any process or system in the Trust to promote cost-effective prescribing. Among these, $44 \%$ reported pharmacists review, $15 \%$ reported $\mathrm{BNF}$ distribution, $11 \%$ reported drug formularies, another $11 \%$ reported the Drugs and Therapeutic Committee, a further $11 \%$ reported consultant review and $8 \%$ reported guidelines (Trust and NICE) as ways of improving cost-effective prescribing.

\section{Discussion}

The main finding of this study is that the awareness and knowledge of cost-effective prescribing are unsatisfactory among psychiatrists working in the Trust. The result is consistent with a recent meta-analysis on this topic ${ }^{7}$ and reflects a lack of concern in this area of clinical practice. Other possible reasons for the lack of awareness could be poor education about the price of drugs and cost-effective prescribing patterns, information overload in doctors or a different perspective regarding cost-effective prescribing.

In the UK, patients only have to pay for the cost of the prescription and not the total cost of the drug. The mental health trusts, however, have to pay the total drug cost. Psychiatrists may initiate drug treatment and GPs then tend to continue with the same choice of drug. Therefore, greater consideration to the choice and cost of drug, particularly on initiating a treatment, affects the overall NHS budget and thereby may influence the long-term availability of certain drugs for our patients. Overpricing clozapine, haloperidol, SSRIs and lithium while underpricing quetiapine raises doubts regarding doctors' awareness of the facts that newer drugs are generally more expensive than older drugs and are 
backed by intensive marketing strategies. Moreover, newer drugs are not always cost-effective. There is a need to develop strategies that can improve the awareness and knowledge of doctors in this increasingly important area of our practice. Doctors are not always unwilling to consider the cost of the drugs they prescribe. If anything, a Canadian study ${ }^{8}$ has shown that doctors were sensitive to the cost of drugs when provided with their costs and reduced expensive prescribing for third-party covered drug as well. Interestingly, they did choose higher-cost drugs for patients with third-party coverage initially when the drug costs were not provided. Also, a qualitative UK study showed that doctors give considerable thought to the cost in their prescribing decisions in the context of ensuring quality of care. ${ }^{9}$

The Department of Health document Our Health, Our Care, Our Say: A New Direction for Community Services ${ }^{10}$ encourages giving more choice to individuals regarding their care. Should this imply that doctors need to inform patients regarding the cost of drugs when helping them to make decisions? The clinicians might well be in unchartered waters when this information regarding costs and patient choice is introduced into day-to-day clinical practice. This is likely to have implications for the doctor-patient relationship and a significant impact on the NHS budget.

In an attempt to reduce drug costs without any detriment to patient care, an action plan was formulated and approved by the Drugs and Therapeutics Committee of the North East London Foundation Trust. The Trust psychotropic drug formulary will be distributed to all doctors and nurse prescribers. A copy of the BNF will continue to be routinely delivered to all prescribers and wards. Additionally, it is important to draw attention to the fact that the BNF is now available online with up-to-date prices and is accessible to all. Information about costeffective prescribing will be emphasised at trainees' induction, academic meetings, ward rounds, supervision and pharmacist reviews. The Drugs and Therapeutics Committee will develop a practical one-page aide memoire providing brief information comparing the price of the most commonly prescribed drug from each psychotropic group. The aide memoire will target prescribing individuals or areas, i.e. attach to the BNF, drugs trolley, clinical rooms, etc.

This study has the following limitations. It was based on a sample collected from the North East London Foundation Trust and gives information only about this trust. As this study has not been carried out before, there were no local, regional or national standards to compare our results with, and hence our benchmarking standards were agreed by consensus. The evaluation instrument has only face validity. The results could have been influenced by volunteer bias. Only $54 \%$ of consultant and $43 \%$ of associate specialist grades completed this survey and these groups are the most senior prescribers in the Trust. In addition, responses to the survey could have been different to actual practice. Table 1 shows the distribution by grade of all the doctors who participated in the service evaluation. However, the service evaluation did not set out to find the prescribing pattern of the whole team and as such the data regarding specific teams was not collected and analysed. Similarly, we did not analyse the magnitude of the misestimates of drug costs in terms of cash value. These issues need to be explored further in future research papers on this topic.

Consideration also needs to be given to the factors that influence the spread of change in healthcare systems. ${ }^{11}$ Our service evaluation shows that doctor's knowledge and awareness in this important area of clinical practice is poor, with existing measures such as routine distribution of the BNF and providing easy access to NICE guidelines in themselves insufficient to affect prescribing practice. Promoting a culture where the cost of drugs is more comfortably and readily considered when prescribing is essential. A relatively modest change in practice is all that may be needed. Berwick notes that for the spread of change to occur there is a tipping point around $15-20 \%$ adoption, after which it becomes difficult to stop the spread of change. ${ }^{11}$ In the current climate of dramatic cuts in the NHS budget, it would seem that the time for such a change in prescribing culture has come.

\section{About the authors}

Dhananjay Kumar Singh is a Specialty Trainee (ST5) in the Barts and the London Training Scheme. Shakil Khawaja is a Consultant Psychiatrist at the North East London Foundation Trust. Ishaq Pala is a Core Trainee (CT3) in the Barts and the London Training Scheme. Jaleel Khaja is a Locum (Staff Grade) in the South London and Maudsley NHS Foundation Trust. Ray Krishnanu is a Core Trainee (CT2) in the Barts and the London Training Scheme. Heather Walker is the Chief Pharmacist at the North East London Foundation Trust. Julian Bustin is a Specialty Registrar in the Barts and the London Training Scheme, London, UK.

\section{References}

1 National Audit Office. Prescribing Cost in Primary Care. National Audit Office, 2007 (http://www.nao.org.uk/publications/0607/ prescribing_costs_in_primary_c.aspx).

2 National Institute for Health and Clinical Excellence. Guidance on the Use of Newer (Atypical) Antipsychotic Drugs for the Treatment of Schizophrenia. Health Technology Appraisal Guidance 43. NICE, 2002.

3 National Institute For Health and Clinical Excellence. Zaleplon, Zolpidem and Zopiclone for the Short Term Management of Insomnia. Health Technology Appraisal Guidance 77. NICE, 2004.

4 National Institute For Health and Clinical Excellence. Donepezil, Galantamine, Rivastigmine (review) and Memantine for the Treatment of Alzheimer's Disease (amended). Health Technology Appraisal Guidance 111 NICE, 2007.

5 British Medical Association and Royal Pharmaceutical Society of Great Britain. British National Formulary (54th edn) (September issue). BMJ Books and Pharmaceutical Press, 2007.

6 Bustin J, Cassone J, Figueroa S. Mental health residents: knowledge on prices and availability of drugs they prescribe [in Spanish]. Vertex 2002; 13: 9-15.

7 Allan G, Lexchin J, Wiebe N. Physician awareness of drug cost: a systematic review. PLoS Medicine 2007; 4: e283.

8 Hux J, Naylor D. Drug prices and third party payment, do they influence medical selection. Pharmacoeconomics 1994; 5: 343-50.

9 Prosser H, Walley T. A qualitative study of GP's and PCO stakeholder's view on the importance and the influence of cost on prescribing. Sco Sci Med 2005; 60: 1335-46

10 Department Of Health. Our Health, Our Care, Our Say: A New Direction for Community Services. Department Of Health, 2006 (http:// www.dh.gov.uk/en/Publicationsandstatistics/Publications/ PublicationsPolicyAndGuidance/DH_4127453).

11 Berwick D. Sauerkraut, sobriety, and spread of change. In Escape Fire: Designs for the Future of Health Care: 98-125. Jossey-Bass, 2004. 